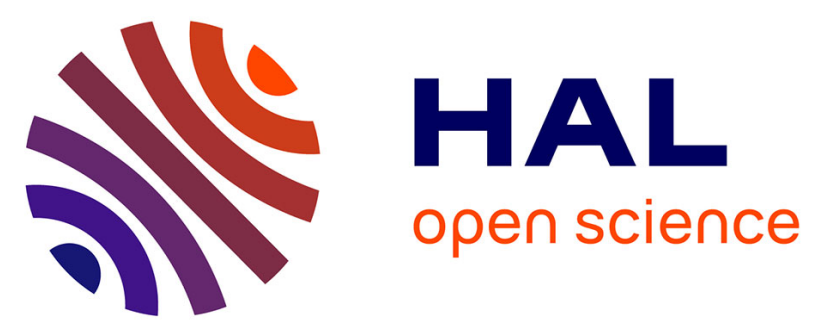

\title{
Stability and thermoelectric performance of doped higher manganese silicide materials solidified by RGS (ribbon growth on substrate) synthesis
}

Pierre-Yves Pichon, Pierre Berneron, Joshua Levinsky, Arjan Burema, Graeme Blake, David Berthebaud, Stéphanie Gascoin, Franck Gascoin, Sylvie Hebert, Jonas Amtsfeld, et al.

\section{To cite this version:}

Pierre-Yves Pichon, Pierre Berneron, Joshua Levinsky, Arjan Burema, Graeme Blake, et al.. Stability and thermoelectric performance of doped higher manganese silicide materials solidified by RGS (ribbon growth on substrate) synthesis. Journal of Alloys and Compounds, 2020, 832, 10.1016/j.jallcom.2020.154602 . hal-03006602

\section{HAL Id: hal-03006602 https://hal.science/hal-03006602}

Submitted on 16 Nov 2020

HAL is a multi-disciplinary open access archive for the deposit and dissemination of scientific research documents, whether they are published or not. The documents may come from teaching and research institutions in France or abroad, or from public or private research centers.
L'archive ouverte pluridisciplinaire $\mathbf{H A L}$, est destinée au dépôt et à la diffusion de documents scientifiques de niveau recherche, publiés ou non, émanant des établissements d'enseignement et de recherche français ou étrangers, des laboratoires publics ou privés. 


\section{Journal Pre-proof}

Stability and thermoelectric performance of doped higher manganese silicide materials solidified by RGS (ribbon growth on substrate) synthesis

Pierre-Yves Pichon, Pierre Berneron, Joshua Levinsky, Arjan Burema, Graeme Blake, David Berthebaud, Stéphanie Gascoin, Franck Gascoin, Sylvie Hebert, Jonas

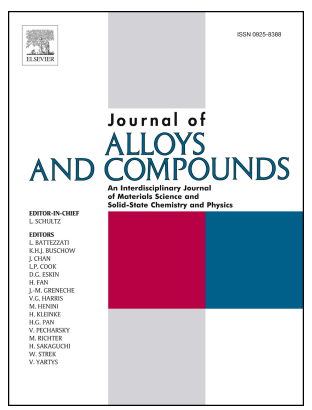
Amtsfeld, Tijmen Hommels, Mark Huijben, Johannes de Boor, Eckhard Müller, Christelle Navone, Axel Schönecker

PII: S0925-8388(20)30965-8

DOI: https://doi.org/10.1016/j.jallcom.2020.154602

Reference: JALCOM 154602

To appear in: Journal of Alloys and Compounds

Received Date: 29 November 2019

Revised Date: 20 January 2020

Accepted Date: 29 February 2020

Please cite this article as: P.-Y. Pichon, P. Berneron, J. Levinsky, A. Burema, G. Blake, D. Berthebaud, Sté. Gascoin, F. Gascoin, S. Hebert, J. Amtsfeld, T. Hommels, M. Huijben, J. de Boor, E. Müller, C. Navone, A. Schönecker, Stability and thermoelectric performance of doped higher manganese silicide materials solidified by RGS (ribbon growth on substrate) synthesis, Journal of Alloys and Compounds (2020), doi: https://doi.org/10.1016/j.jallcom.2020.154602.

This is a PDF file of an article that has undergone enhancements after acceptance, such as the addition of a cover page and metadata, and formatting for readability, but it is not yet the definitive version of record. This version will undergo additional copyediting, typesetting and review before it is published in its final form, but we are providing this version to give early visibility of the article. Please note that, during the production process, errors may be discovered which could affect the content, and all legal disclaimers that apply to the journal pertain.

C 2020 Published by Elsevier B.V. 


\title{
Stability and thermoelectric performance of doped Higher Manganese Silicide materials solidified by RGS (Ribbon Growth on Substrate) synthesis
}

Pierre-Yves Pichon* ${ }^{* a}$, Pierre Berneron ${ }^{\mathrm{a}}$, Joshua Levinsky ${ }^{\mathrm{b}}$, Arjan Burema ${ }^{\mathrm{b}}$, Graeme Blake ${ }^{\mathrm{b}}$, David Berthebaud $^{\mathrm{c}}$, Stéphanie Gascoin ${ }^{\mathrm{c}}$, Franck Gascoin ${ }^{\mathrm{c}}$, Sylvie Hebertc ${ }^{\mathrm{c}}$, Jonas Amtsfeld ${ }^{\mathrm{d}}$, Tijmen Hommels $^{\mathrm{d}}$, Mark Huijben ${ }^{\mathrm{d}}$, Johannes de Boor ${ }^{\mathrm{e}}$, Eckhard Müller ${ }^{\mathrm{e}, \mathrm{g}}$, Christelle Navone ${ }^{\mathrm{f}}$, Axel Schönecker ${ }^{\mathrm{a}}$

*corresponding author: pichon@ @rgsdevelopment.nl

List of co-authors e-mail addresses (in order of appearance in the author list): berneron@ rgsdevelopment.nl; j.levinsky@rug.nl; a.a.burema@rug.nl; g.r.blake@ rug.nl; David.BERTHEBAUD@cnrs.fr; stephanie.gascoin@ensicaen.fr; franck.gascoin@ensicaen.fr; sylvie.hebert@ensicaen.fr; jonas.amtsfeld@gmail.com; tijmenhommels@ hotmail.com m.huijben@utwente.nl; johannes.deboor@dlr.de; Eckhard.Mueller@dlr.de; christelle.navone@ cea.fr; schonecker@rgsdevelopment.nl

${ }^{a}$ RGS Development B.V., Bijlestaal 54a, 1721 PW Broek op Langedijk, Netherlands

bernike Institute for Advanced Materials, University of Groningen, Nijenborgh 4, 9747AG Groningen, Netherlands

${ }^{\mathrm{c}}$ Normandie Univ, ENSICAEN, UNICAEN, CNRS, CRISMAT, UMR6508, 14000 Caen, France

${ }^{\mathrm{d}}$ MESA+ Institute for Nanotechnology, University of Twente, 7500 AE Enschede, Netherlands

${ }^{\mathrm{e}}$ German Aerospace Center, Institute of Materials Research, Linder Höhe, 51147 Köln, Germany

${ }^{\mathrm{f}}$ CEA, LITEN, 17 rue des Martyrs, BP166, 38042 Grenoble, France

${ }^{g}$ Justus Liebig University Gießen, Institute of Inorganic and Analytical Chemistry, 35392 Gießen, Germany

\section{Keywords}

Thermoelectric materials; crystal growth; microstructure; crystal structure; electrical transport

\begin{abstract}
Large scale deployment of thermoelectric devices requires that the thermoelectric materials have stable electrical, thermal and mechanical properties under the conditions of operation. In this study we examine the high temperature stability of higher manganese silicide (HMS) materials prepared by the RGS (ribbon growth on substrate) technique. In particular we characterize the effect of element substitution on the structural and electrical changes occurring at the hot side of temperatures of thermoelectric devices relevant to this material $\left(600^{\circ} \mathrm{C}\right)$. Only by using suitable substitution $(4 \%$ vanadium at the $\mathrm{Mn}$ site) can we obtain temperature-independent structural parameters in the range $20^{\circ} \mathrm{C}-600^{\circ} \mathrm{C}$, a condition that results in stable electrical properties. Additionally, we show that $4 \%$ vanadium substitution at the $\mathrm{Mn}$ site offers the best thermoelectric figure of merit among the different compositions reported here with $Z T_{\max }=0.52$, a value comparable to the state of the art for HMS materials. Our analysis suggests that ionized impurity scattering is responsible for the better performance of this material.
\end{abstract}




\section{Introduction}

The higher manganese silicide (HMS, $\mathrm{MnSi}_{\gamma}$ ) phases are the most silicon-rich materials of the $\mathrm{Mn}-\mathrm{Si}$ alloys. HMS materials are promising candidates for the deployment of large scale, costeffective thermoelectric generators operating up to $600^{\circ} \mathrm{C}$ thanks to their low raw material cost and good thermoelectric performance. The thermoelectric figure of merit $(Z T)$ characterizes the effectiveness of a material to transform a heat flow into electrical power. It is defined as $Z T=S^{2} \sigma T / \kappa$, where $S$ is the Seebeck coefficient, $\sigma$ is the electrical conductivity, $\kappa$ is the thermal conductivity, and $T$ is the absolute temperature. Competitive $Z T$ values in the range 0.5-0.7 have been measured on doped HMS materials synthesized by different techniques, which makes them technologically relevant for integration into thermoelectric devices[1-3], possibly in combination with n-type silicides (SiGe alloys, $\left.\mathrm{Mg}_{2}(\mathrm{Si}, \mathrm{Ge}, \mathrm{Sn})[4,5]\right)$. Recently, supersaturated HMS materials synthesized by melt spinning achieved significantly improved performance with $Z T$ values of about 1 for p-type Re-doped HMS [68]. Until now most of the work on thermoelectric materials has focused on increasing ZT. However, an important condition for successful large-scale deployment of high temperature thermoelectric devices is the stability of the thermoelectric properties at the relevant temperature and atmospheric conditions. The first objective of this work is to systematically characterize the high temperature electrical stability of HMS materials produced by the Ribbon Growth on Substrate (RGS) production process. We aim at clarifying the relations between electrical stability, doping type and structural evolution under high temperature module operation conditions. In this article we also demonstrate a significant improvement of both electrical stability and $Z T$ for vanadium-doped HMS associated with ionized impurity scattering of the charge carriers and subsequent superior electrical and thermal properties.

The HMS phases belong to the family of so-called chimney-ladder structures: a [Mn] 'chimney' subsystem and a [Si] 'ladder' subsystem having a common $a$-axis but different stacking periods in the $c$ direction, hence different subsystem $c$-axis lengths $c_{M n}$ and $c_{S i}$. Examination of the crystal structure shows that $\gamma=c_{M n} / c_{S i}$. If $\gamma$ is an irrational number the structure is incommensurate and cannot be described using a single overall lattice parameter in the $c$ direction: this motivates the structural characterization of HMS in terms of two separate subsystems or by the (3+1) dimensional superspace approach[9]. More recently another approach was proposed to model the XRD spectrum of the HMS structure by including significant defects in the Si sublattice [10]. The valence electron count $(V E C)$ concept is useful to describe the thermodynamic stability of chimney-ladder phases and to estimate the charge carrier density of these materials from the structural parameter $\gamma$ [11-14]. The VEC is the number of valence electrons per transition metal element (here $\mathrm{Mn}$ ). For unsubstituted $\mathrm{MnSi}_{\gamma}$, $V E C=7+4 \gamma$. For the general case of substituted HMS it can be written as:

$$
V E C=7(1-x)+V_{M e} x+\left(4(1-y)+V_{S} y\right) \gamma
$$

Here $x$ and $V_{M e}$ are the proportion and number of valence electrons of the element $M e$ that substitutes Mn (e.g., $V_{V}=5$ ); $y$ and $V_{s}$ are the proportion and number of valence electrons of the element that substitutes $\mathrm{Si}$ (e.g., $\left.V_{A l}=3 ; V_{G e}=4\right)$. Furthermore, the charge carrier density can be estimated by:

$$
[p]=\frac{4(14-V E C)}{v}
$$

In equation (2) $\mathcal{V}$ is the volume of the Mn subcell. Here we consider only the case where $V E C<14$ (ptype materials). In general, chimney-ladder phases are stabilized for a VEC count of 14 [11,12]. Therefore, according to equation (2) stable chimney ladder phases should be charge neutral: in fact thermodynamic stability relates to the opening of a bandgap around the chemical potential. For the HMS phase the stoichiometry $\mathrm{Mn}_{4} \mathrm{Si}_{7}(\gamma=1.75)$ should be preferred, however structural analysis has shown that compounds with lower $\gamma$ are actually synthesized, conferring to this material an intrinsic ptype character. Kawasumi et al. identified $\mathrm{Mn}_{15} \mathrm{Si}_{26}$ as the stoichiometry of undoped-HMS solidified by the Bridgman method[15] and also measured a hole density of about $2 \times 10^{27} \mathrm{~m}^{-3}$ in the room 
temperature-to $600 \mathrm{~K}$ range [15], as predicted by equation (2) $\left([p]=1.98 \times 10^{27} \mathrm{~m}^{-3}\right)$. Early works identified $\mathrm{Mn}_{15} \mathrm{Si}_{26}(\gamma=1.733), \mathrm{Mn}_{11} \mathrm{Si}_{19}(\gamma=1.727)$, and $\left.\mathrm{Mn}_{27} \mathrm{Si}_{47}(\gamma=1.741)\right)$ as commensurate structures[15-17], while more recent studies report intermediate $\gamma$ values, interpreting these as either incommensurate, incorporating defects at the Si sites, or comprising domains of different $\gamma$ values $[9,10,18-20]$.

Several studies have shown that undoped HMS can undergo solid-solid phase transitions in the temperature range useful for thermoelectric generation. For example a comparative study of Differential Thermal Analysis (DTA), Differential Scanning Calorimetry (DSC) and High Temperature powder X-Ray Diffraction (HTXRD) showed that solid-solid phase transformations occur upon heating[21]. These transitions are primarily due to a temperature-dependence of the $\gamma$ ratio: another HTXRD study of undoped HMS showed that the expansion rates of the Si and Mn sublattices in the $c$ direction are different upon heating. As a result $\gamma$ increases and the structure expels $\mathrm{Mn}$, resulting in $\mathrm{MnSi}$ precipitation in the form of layered precipitates parallel to the $a-b$ crystallographic plane [13]. In this study the authors suggest that the long-term stability of the material may be compromised due to cracks occurring at the HMS-MnSi interface. Cracking issues in the $a-b$ plane have been a general concern for HMS solidified from the liquid[22], the cause being the lattice mismatch between the HMS phase and the MnSi phase precipitated during cooling from the melting point[23,24]. Finally, another HTXRD study showed that the addition of alloying elements at the Mn or Si site can significantly influence the relative expansion coefficient of the sublattices, i.e. the $\gamma(T)$ characteristic. For example, in the case of Ge doping $\gamma$ is stable between room temperature and 600 ${ }^{\circ} \mathrm{C}[19]$. This suggests that appropriate doping may stabilize the HMS phase in the temperature range of interest. Notably, above a critical Ge concentration the layered MnSi precipitates disappeared in crystals grown by the Czochralski technique[22].

Despite several literature reports on the HMS phases show the occurrence of a solid-solid reactions in the temperature range of interest for thermoelectric applications, very little information is available on the stability of the thermoelectric properties, and on their relations with the synthesis technique, the concentration of a particular substitution element, and the corresponding $\gamma(T)$ characteristics. Most papers have reported the thermoelectric properties of as-synthesized materials, without demonstrating that the materials are thermodynamically and/or electrically stable. The motivation of this work is to bridge the gap between stability studies and performance studies of the HMS materials. In this framework, here we examine the effects of doping on the (micro)structural evolution of HMS and on the changes in thermoelectric properties at high temperatures. We study the effect on the thermoelectric properties of maintaining the materials at a temperature of $600^{\circ} \mathrm{C}$ in ambient atmosphere. Based on a review of the literature, we expected that synthesis techniques involving relatively fast cooling such as RGS (about $2 \mathrm{~K} / \mathrm{sec}$ above $500{ }^{\circ} \mathrm{C}$ ) may result in metastable $\gamma$ after synthesis. Upon further high temperature exposure in devices this might result in $\mathrm{MnSi}$ precipitation, cracking, and changes in [p]. Therefore, we use HTXRD to characterize $\gamma(T)$ in the room temperature-to $600^{\circ} \mathrm{C}$ range and to explain changes in the microstructure, in the charge carrier density and in the mobility upon aging. This work is reported in section 3.1.

Since the doping type and level are key parameters affecting the $\gamma(T)$ characteristics, we chose a set of nominal compositions based on promising materials reported so far. $\mathrm{Mn}\left[\mathrm{Si}_{0.9955} \mathrm{Al}_{0.0045}\right]_{1.73}$ was chosen because of the good thermoelectric properties already reported[1]. We selected $\mathrm{Mn}\left[\mathrm{Si}_{0.991} \mathrm{Ge}_{0.009}\right]_{1.73}$ because of the reported effects of $\mathrm{Ge}$ on $\gamma(T)$ and MnSi precipitation $[22,25,26]$. Mn substitution by Re was also chosen because it yields a state of the art $Z T$ value [6], but we opted for a lower doping amount of $\left[\mathrm{Mn}_{0.97} \mathrm{Re}_{0.03}\right] \mathrm{Si}_{1.73}$ to avoid Re silicide formation during synthesis with our RGS technique. The substitution of $\mathrm{V}$ for Mn above $2 \%$ was recently shown to suppress the formation of MnSi precipitates while highly disordered strained Si domains appeared instead [27,28]. Therefore, we included $\left[\mathrm{Mn}_{0.98} \mathrm{~V}_{0.02}\right] \mathrm{Si}_{1.73}$ and $\left[\mathrm{Mn}_{0.96} \mathrm{~V}_{0.04}\right] \mathrm{Si}_{1.73}$ as nominal compositions. Throughout the following discussion the materials will be referred to by the percentage of substituted elements, such as '4\% V-HMS' for $\left[\mathrm{Mn}_{0.96} \mathrm{~V}_{0.04}\right] \mathrm{Si}_{1.73}$. While characterising these materials we noticed that $\mathrm{V}$ - 
doped materials exhibit significantly different electrical properties compared to the others. A comparative analysis of the thermoelectric performance of the different materials is the subject of section 3.2.

\section{Materials and methods}

\subsection{Synthesis}

The materials were synthesized by solidification from the melt using the RGS process. This solidification technique consists in moving a flat mould band (kept at a temperature several hundred Celsius lower than the melting point) under the liquid melt, which is contained in a continuously refilled reservoir. The liquid is solidified at a growth rate between 0.1 and $1 \mathrm{~mm} . \mathrm{s}^{-1}$ resulting in a sheet of typical thickness $0.5 \mathrm{~mm}$ grown on the mould. The solidified semiconductor sheet detaches upon cooling, can be transferred out of the process chamber and the mould can be reused. A detailed description of the process is given in $[29,30]$. The solidification velocity is intermediate between that of large batch growth processes (the Czochralski, floating zone, directional solidification and Bridgman techniques have a solidification velocity of about $10^{-5}-10^{-4} \mathrm{~m} \cdot \mathrm{s}^{-1}$ ) and non-equilibrium processes such as spin casting with a solidification velocity of several $\mathrm{m} \cdot \mathrm{s}^{-1}$. Therefore, RGS materials have unique (micro)structures and electrical properties. This process is interesting from a technological point of view as it allows for HMS thermoelectric legs of controlled dimensions to be produced in one step and at rates compatible with the large-scale deployment of thermoelectric generators.

\subsection{Characterization}

The stability of the materials was tested under two types of conditions. In a first series of tests the Seebeck coefficient and the electrical conductivity of as-solidified samples were measured as a function of time at $600{ }^{\circ} \mathrm{C}$ in inert gas (12 to 45 hours). Such measurements were done routinely at University of Groningen and selected samples were measured at CEA and University of Twente for confirmation. In a second series of tests the samples were subjected to annealing steps at $600{ }^{\circ} \mathrm{C}$ for 10 hours, then for 100 hours in air. These testing conditions are more relevant to practical applications since a thermoelectric material that is usable in oxidative environments opens the door to more costeffective module designs compared to, e.g. encapsulated module configurations. The Seebeck coefficient and electrical conductivity were measured at room temperature on each sample in the asgrown state, after 10 hours of annealing and after 10+100 hours of annealing. Additional samples were undergoing the same annealing sequence and were analysed by Hall measurements, microstructure analysis, HTXRD and thermoelectric performance evaluation. For all samples where the Seebeck coefficient and/or electrical conductivity were measured (including Hall samples), one surface of the RGS-cast HMS sheet samples was ground to reduce thickness inhomogeneities.

Note that our analysis plan allowed for cross-checking of the measurements performed at three different laboratories for the room temperature Seebeck coefficient and at four different laboratories for the electrical conductivity. We verified a posteriori that the error bars indeed overlap.

\subsubsection{Stability: structure and microstructure}

Samples on which the microstructure was studied were embedded in epoxy resin and polished (last step using a $1 \mu \mathrm{m}$ diamond suspension) for analysis by optical microscopy and Scanning Electron Microscopy (SEM). In order to reveal the presence of MnSi precipitates the polished cross-sections were submitted to a dilute HF etching step (HF:H2O=5:95, typically $10 \mathrm{~s}$ ). SEM analysis was performed using a Hitachi SU70 electron microscope with an acceleration voltage of $15 \mathrm{kV}$ and a working distance of $15 \mathrm{~mm}$. Energy dispersive X-ray spectroscopy (EDX) was carried out on some samples to identify the different phases present and evaluate the composition homogeneity.

High resolution powder or bulk surface X-ray diffraction data were collected using a Bruker D8 Advance Vario 1 two-circle diffractometer ( $\theta-2 \theta$ Bragg-Brentano mode) using $\mathrm{Cu} \mathrm{K} \alpha$ radiation 
$\left(\lambda=1.540598 \AA\right.$ A ) with a Ge ( $\left.\begin{array}{lll}1 & 1 & 1\end{array}\right)$ monochromator (Johansson type) and a Lynx Eye detector. Hightemperature experiments were carried out with an Anton Paar furnace HTK1200N; the temperature was regulated within $\pm 3 \mathrm{~K}$. Data were collected over the angular range $10 \leq 2 \theta\left(^{\circ}\right) \leq 95$ under air, measuring for $1 \mathrm{~s}$ at each angular increment of $0.0157^{\circ}(1.5 \mathrm{~h} / \mathrm{scan})$. XRD patterns were recorded using the following temperature profile: a pattern at room temperature; one every $100 \mathrm{~K}$ from $373 \mathrm{~K}$ to $873 \mathrm{~K}$ (heating rate of $30 \mathrm{~K} / \mathrm{min}$ ); one every $100 \mathrm{~K}$ from $873 \mathrm{~K}$ to $373 \mathrm{~K}$ (cooling rate of $30 \mathrm{~K} / \mathrm{min}$ ); one at room temperature after cooling. The Le Bail method was employed to refine the lattice parameters and modulation vectors. Refinements were performed in the $(3+1)$-dimensional superspace group I41/amd(00g)00ss using the JANA2006 software package[31].

\subsubsection{Stability: thermoelectric properties}

The Seebeck coefficient and electrical conductivity were measured simultaneously using a Linseis LSR-3 apparatus at the University of Groningen. To measure the Seebeck coefficient a temperature gradient of $30 \mathrm{~K}$ was applied across the sample and the induced voltage was measured at a constant current. The resistivity was measured using a four-point probe configuration where all contact points are aligned along a vertical line. To characterize the evolution of the electrical resistivity and Seebeck coefficient at elevated temperature, the samples were held at $600{ }^{\circ} \mathrm{C}$ for 14 hours under a protective helium atmosphere to prevent oxidation.

For the samples annealed in air at $600{ }^{\circ} \mathrm{C}$ the Seebeck coefficient and electrical conductivity were measured at room temperature on a custom-made test bench at RGS Development B.V. An HF dip was performed before the measurement to remove the oxide layer grown during the annealing step. The electrical conductivity was measured on a four-point probe system using an electrical current of 0.5 A over the (measured) sample cross-section area of approximately $0.4 \times 15 \mathrm{~mm}^{2}$. The error of the electrical conductivity is estimated to be less than $8 \%$, the highest error contribution coming from thickness variations. The Seebeck coefficient was evaluated by measuring the voltage drop generated by a temperature difference of $8 \mathrm{~K}$ around room temperature. The temperature difference was applied using commercially available Peltier elements and measured using type T thermocouples.

To evaluate the change in charge carrier density and mobility upon annealing, Hall measurements were carried out at CRISMAT labs on as-solidified samples and samples annealed for 10 hours at $600{ }^{\circ} \mathrm{C}$ in air. The measurements were performed in a PPMS system from Quantum Design, where the electrical contacts were soldered with indium on samples with a shape $5 \times 5 \times 0.4$ $\mathrm{mm}$. The carrier density was extracted from Hall effect measurements from $5 \mathrm{~K}$ to $300 \mathrm{~K}$ considering as a first approximation a simple single band model, to compare with the results from the literature $[1,28]$. Electrical resistivity was measured using the van der Pauw geometry. Note that the measurements were performed in plane, and no noticeable anisotropy was detected during the Van der Pauw experiments. This is consistent with EBSD analysis which showed that the out of plane direction corresponds to the $\mathrm{c}$ axis direction. From both sets of measurements, the mobility could be extracted. For the resistivity the main source of error comes from the thickness determination, with a maximal error of $\sim 8 \%$ except for the $\mathrm{V}$-doped materials where the error is $\sim 12 \%$ due to thinner solidified samples, and from the contact size and misalignment, estimated to be $\sim 5 \%$. The intrinsically high charge carrier density of HMS materials makes the measurement accuracy particularly sensitive to errors in the measurement of the slope of the Hall resistance curves $\mathrm{R}(\mathrm{H})$. All the carrier concentrations determined here are extracted from linear $\mathrm{R}(\mathrm{H})$ curves, the uncertainty in the linear least squares fit being less than $0.5 \%$.

\subsubsection{Thermoelectric performance}

Samples were measured at DLR to evaluate their thermoelectric performance. The samples were polished before the measurements to decrease the surface roughness and improve the electrical contacts. The electrical conductivity $\sigma$ and the Seebeck coefficient $S$ of the samples were measured concurrently using a custom-built setup. Setup details and a detailed description of the data analysis for the Seebeck coefficient measurements can be found in published works[32,33]. Due to the small thickness and the sometimes uneven surface, the uncertainty for the electrical conductivity is estimated to be $10 \%$, while the uncertainty for the Seebeck coefficient is $5 \%$. The measurement data were 
acquired both during heating up and during cooling down. The thermal diffusivity $(\alpha)$ of the samples was obtained using a Netzsch LFA 467HT apparatus. The density $\rho$ was determined using Archimedes' method, while the heat capacity $C_{P}$ was measured using a Netzsch DSC 404 . The thermal conductivity $(\kappa)$ was obtained using the relation $\kappa=\alpha \rho C_{P}$ and the measurement accuracy is estimated to be $10 \%$, the uncertainty stemming mainly from the $C_{P}$ measurement and the thickness sensitivity of the LFA measurement. Note that for practical reasons of sample geometry the conductivity (and Seebeck coefficient) are determined in-plane, while thermal conductivity is determined cross-plane. Therefore the ZT results reported here should be seen as best estimates, since one cannot exclude possible anisotropic effects.

\section{Results and Discussion}

\subsection{Stability}

\subsubsection{Stability of the electrical properties}

Figure1 shows measurements of the Seebeck coefficient and electrical conductivity as a function of time at $600^{\circ} \mathrm{C}$ for $4 \% \mathrm{~V}-\mathrm{HMS}, 0.9 \% \mathrm{Ge}-\mathrm{HMS}, 0.45 \% \mathrm{Al}-\mathrm{HMS}$, and $3 \% \mathrm{Re}-\mathrm{HMS}$. The measurements show that the electrical resistivity increases by between $6 \%$ and $20 \%$ during the $12 \mathrm{~h}$ of measurement time for the $\mathrm{Ge}, \mathrm{Al}$ and Re doped samples. Note that for these samples the electrical resistivity still continues to increase at the end of the test. In contrast, the electrical resistivity of $4 \% \mathrm{~V}$ HMS is found stable over time and does not vary within the measurement error. Changes in the Seebeck coefficient, if any, are at most $2 \%$ based on data analysis after noise filtering, which is relatively small compared to changes in the resistivity. Our data show that the $4 \% \mathrm{~V}$-HMS has stable electrical properties at $600^{\circ} \mathrm{C}$, a typical hot-side thermoelectric module temperature. We confirmed this observation by measuring additional samples at different laboratories for up to 45 hours. Figure S1 in the supplementary information shows the evolution of the electrical properties of $4 \% \mathrm{~V}-\mathrm{HMS}$ and $0.45 \% \mathrm{Al}-\mathrm{HMS}$ as a function of time. Figure 2 summarizes these results by showing a comparison of the high temperature power factor of different materials as-solidified and after 10 hours exposure to $600^{\circ} \mathrm{C}$.

Since we are interested in the material stability under atmospheric conditions, we also measured the changes of the room temperature electrical conductivity and Seebeck coefficient for samples that had undergone successive annealing for $10 \mathrm{~h}$ and $100 \mathrm{~h}$ in air at $600{ }^{\circ} \mathrm{C}$. The effect of the two annealing steps on the room temperature electrical properties is shown in figure 3. Except for $4 \% \mathrm{~V}-\mathrm{HMS}$ where the electrical properties do not change, the electrical conductivity of all materials (including 2\%V-HMS) decreases by $20-25 \%$ after $10+100$ hours of annealing, while the change in the Seebeck coefficient is relatively smaller. Figure 2 and figure 3 show that the decrease in electrical conductivity on annealing results in a significant reduction of the power factor $S^{2} \sigma$, with the exception of $4 \% \mathrm{~V}$-HMS. Note that the material with the highest power factor before thermal treatment is the Gedoped material but after annealing the V-doped materials have the best electrical properties.

Additionally, we conclude that the testing atmosphere is likely not the most influential parameter to explain the changes in electrical properties, and that a minimum vanadium concentration is required to stabilize the electrical conductivity.

\subsubsection{Structure and microstructure analysis}

The purity of the $2 \% \mathrm{~V}-\mathrm{HMS}, 4 \% \mathrm{~V}-\mathrm{HMS}$, and $0.45 \% \mathrm{Al}-\mathrm{HMS}$ materials and the absence of any significant volume fraction of secondary phases were checked by XRD measurements on powder samples derived from as-solidified materials. The XRD patterns are shown in figure 4 and demonstrate the absence of significant amounts of $\mathrm{MnSi}$, Al-rich or $\mathrm{V}$-rich phases. The variation of peak intensity is due to preferential orientation effects. All peaks could be indexed as HMS phase with superspace group I41/amd( $00 \gamma) 00$ ss according to the composite structural model suggested by Miyazaki et al.[34]. According to research on the $\mathrm{Mn}-\mathrm{Si}$-Al system, excess Al-doping would result in the formation of $\mathrm{Al}$ rich phases with about 30 at $\% \mathrm{Al}$ (C40 or $\mathrm{C} 54$ phase) [35,36], which are not observed in our case. This confirms that the chosen $\mathrm{Al}$ doping level is below the solubility limit as already demonstrated by Chen et al.[1]. Similarly, we do not observe any signal from $\mathrm{VSi}_{2}$ in $2 \% \mathrm{~V}-\mathrm{HMS}$ and $4 \% \mathrm{~V}-\mathrm{HMS}$, which 
would be the case if we significantly exceeded the solubility limit of V in the HMS phase. However, Miyazaki et al. suggested that the solubility limit of V substituting Mn in HMS is 3\% [27]. As will be discussed later, small amounts of the MnSi phase are present in all materials but could not be detected by the XRD measurements because of their low volume fraction.

All materials (as-grown and annealed) presented in figure 3Figure were analysed by optical microscopy to search for the presence of MnSi. Some samples were also analysed by SEM-EDX to characterize the homogeneity of the dopant distribution in the HMS matrix and to identify secondary phases, if present. The microstructural changes of the $0.45 \% \mathrm{Al}-\mathrm{HMS}$ material are representative of that of the unstable materials ( $\mathrm{Re}, \mathrm{Al}$, and $\mathrm{Ge}$-doped), and therefore only the microstructure analysis of this material is reported here, as compared to that of the stable 4\%V-HMS. A comparison of the optical microscopy features of the as-solidified and annealed materials is shown in figure 5 . The $\mathrm{MnSi}$ phase is identified by different contrast to the bulk due to the effect of the MnSi etching step (this was confirmed by measuring a ratio $[\mathrm{Si}]:[\mathrm{Mn}]=1: 1$ using SEM-EDX). For both materials the presence of a small volume fraction of MnSi dendritic crystals at the surface contacting the mould is noticed. Because of the presence of a peritectic transformation it is expected that solidification starts first by MnSi nucleation at the mould surface and further by dendritic growth, and that later the HMS phase overgrows the MnSi dendrites in a melt slightly enriched in Si [37].

The main feature that distinguishes the as-solidified 4\%V-HMS from the 0.45\%Al-HMS material is the presence of $\mathrm{MnSi}$ layered precipitates in the latter, while these so-called striations are absent from 4\%V-HMS. This observation confirms the general trend observed by other authors[27] on melt-grown V-doped HMS materials, although in contrast to them we did observe a minor amount of striations also in the $2 \% \mathrm{~V}$-HMS. For the Al-doped material the striation defects are concentrated in the centres of the grains and often absent close to the grain boundaries. Local analysis of the composition by EDX showed that the concentration of $\mathrm{Al}$ dopant corresponds to about $0.2 \%$ substitution of $\mathrm{Si}$ in the striated regions and up to more than $1 \%$ substitution closer to the grain boundaries, while the nominal substitution amount is $0.45 \%$. Similarly, we observe that for the Ge-doped materials, higher [Ge] closer to grain boundaries is correlated with the absence of striations there, as also reported by other authors for Ge-doped arc-melted samples[18]. As observed by Vives[18], above a critical Ge or Al concentration MnSi striations do not precipitate during cooling after solidification. As will be discussed later, this is likely due to the stabilization effect of the substituted element on the $\gamma(T)$ characteristic above a critical concentration. In our case the concentration gradients originate from dopant segregation during RGS solidification. Note that the presence of gradients in the concentration of substitution elements imply that our materials are electrically inhomogeneous in the as-solidified state.

The effect of annealing the samples in air at $600{ }^{\circ} \mathrm{C}$ for 10 hours is also shown in figure 5 . We do not observe significant microstructural changes for the 4\%V-HMS, whereas for all the other materials (including the $2 \% \mathrm{~V}-\mathrm{HMS}$ ), we observe a layer of $\mathrm{MnSi}$ at the grain boundaries, as revealed by the dark contrast in the optical microscopy images. Local SEM-EDX analysis confirmed that the stoichiometry of the grain boundary $(\mathrm{GB})$ precipitates is approximately $[\mathrm{Si}]:[\mathrm{Mn}]=1: 1$. The morphology of the striation defects is also different after annealing: striations disappeared completely in some regions or broke down into discontinuous dotted lines. For the as-grown and annealed Aldoped materials, microcracks are often observed along the striations and for the annealed materials voids are present at the MnSi-rich grain boundaries (denoted as 'GB voids' in figure 5). Whether or not these voids are induced by the sample preparation procedure, in both cases we conclude that the interface between HMS and the MnSi phase is mechanically weak due to the significant lattice mismatch [23-25]. Finally it is worth mentioning that the striation defects have an orientation corresponding to a tilt of at most about $30^{\circ}$ with the wafer plane. EBSD measurements confirmed a preferential orientation with the c-axis pointing out of wafer plane. This implies that any anisotropy in the thermoelectric properties, if present, should result in properties in plane different from cross plane. The 4\% V-HMS material is the only one that does not show MnSi precipitation as striations or at grain boundaries in as-grown and annealed samples. This is also the only electrically stable material. We can thus correlate electrical stability with the absence of $\mathrm{MnSi}$ in this material. We are thus able to propose the following two hypotheses: 
1 The formation of MnSi results in microcracks and lowers the charge carrier mobility.

2 The formation of MnSi is the result of structural changes (e.g, the $\gamma$ ratio) in the HMS lattice, which also influences the charge carrier density via equations [1] and [2].

\subsubsection{High temperature structural characterization and Hall measurements}

To clarify hypotheses 1 and 2 we first investigated how the structural parameters of assolidified $0.45 \% \mathrm{Al}-\mathrm{HMS}, 2 \% \mathrm{~V}-\mathrm{HMS}$ and $4 \% \mathrm{~V}$-HMS evolve between room temperature and $600{ }^{\circ} \mathrm{C}$ using HT- powder XRD on as-solidified materials. The samples are the same as for the measurements shown in figure 4. We chose the timescale of the HTXRD measurements and the exposure time at high temperature (total cycle time: 17 hours, time above $500{ }^{\circ} \mathrm{C}: 4.5$ hours) to be a similar order of magnitude to the timescale over which changes in the electrical properties were measured (see figure 1). The complete set of unit cell parameters are plotted in the supplementary information (Figure S2). Figure 6 shows the evolution of $\gamma$ as a function of temperature and measurement sequence. In essence, plotting $\gamma(T)$ is equivalent to characterizing the position of the HMS phase in the temperaturecomposition phase diagram. Figure 6 demonstrates that the HMS phase composition for $0.45 \% \mathrm{Al}-$ HMS and 2\%V-HMS changes during the measurement: the HMS phase is enriched with Si upon heating above about $300{ }^{\circ} \mathrm{C}$, primarily due to a negative expansion coefficient of the silicon sub-lattice in the $c$-direction during heating, i.e. the $\mathrm{Si}$ sub-cell contracts in the $c$-direction while the $\mathrm{Mn}$ subcell expands (see Figure S2, supplementary information). Upon cooling the expansion coefficient of both sub-lattices in the $c$-direction is similar, $\gamma$ is stable and the HMS phase composition remains unchanged.

Kikuchi et al.[13] showed that the expansion coefficient of the Si sublattice in the c-direction is higher than that of the Mn sublattice in the temperature range 500-900 ${ }^{\circ} \mathrm{C}$. This results in the HMS phase becoming more Si-rich upon cooling (see Figure 6) and is in fact the driving force for the formation of MnSi striation precipitates during the cooling of melt-grown HMS crystals as observed by several authors[15,18,38]. We observe MnSi striation precipitates in our as-solidified $0.45 \% \mathrm{Al}$ HMS and $2 \% \mathrm{~V}$-HMS materials, therefore we expect that the $\gamma(T)$ for these materials is similar in shape to that of Kikuchi et al.[13] above $600{ }^{\circ} \mathrm{C}$. However, in our case these materials are likely not stabilized due to the fast cooling rate between the solidification temperature and room temperature in the RGS process (of the order of $2^{\circ} \mathrm{K} / \mathrm{s}$ ). Thus, during synthesis the HMS phase relaxes only partially upon cooling and $\gamma$ is kinetically 'locked' to values lower than the thermodynamically stable value. During the XRD measurement and the annealing tests, the HMS phase stabilizes to higher $\gamma$ values. This explains the changes in $\mathrm{MnSi}$ striation morphology and the appearance of $\mathrm{MnSi}$ at grain boundaries (Figure 5) for the samples that had a similar exposure time at high temperature. In the $4 \% \mathrm{~V}-\mathrm{HMS}$ material, the absence of $\mathrm{MnSi}$ striation precipitates suggests that the composition of the HMS phase solidified at high temperature is stable down to room temperature. This is supported by the temperature-independent $\gamma(T)$ characteristic. These measurements clearly show that stable HMS thermoelectric materials can be obtained by avoiding structural changes between the synthesis temperature and the temperature range of operation in a module. In other words the two sub-lattices should have the same expansion coefficient along the $c$ axis and $\gamma$ should be independent of temperature. The manipulation of $\gamma(T)$ can be achieved by adding substitution elements[19,20], and we have shown that this approach can be used to stabilize the HMS phase over the room temperature-to$600{ }^{\circ} \mathrm{C}$ range. One may further generalise to state that the use of substitution elements that shifts $\gamma$ to (lower) values close to that at the synthesis temperature helps to prevent the precipitation of $\mathrm{MnSi}$ striations. We believe that this also explains the success of using $\mathrm{Ge}$ as a substitution element reported by several researchers, although in our case large [Ge] gradients most likely prevent sufficiently high substitution in the intra-grain regions such that MnSi still precipitates[18,19,25,26].

To examine the relations between changes in the structure and the electrical resistivity, considering as a first approximation a simple single band model, we compared the room temperature charge carrier density estimated by equations [1] and [2] with that measured by the Hall technique, before and after annealing. The results are summarized in Figure 7, which compares the Hall carrier density measured at room temperature on as-solidified materials and on samples annealed for 10 hours at $600{ }^{\circ} \mathrm{C}$ in air with the carrier density estimated from the VEC model using the compositions 
determined from room temperature powder XRD data at the beginning and end of the measurement cycle (data from the samples in Figure 6). In equation (1) we use the nominal substitution levels $x$ and $y$ with the assumption that the solubility limit is not exceeded, as confirmed by the absence of aluminium or vanadium silicides (XRD data, Figure 4). Our Hall concentration data fits very well with that recently published by Miyazaki et al. for V-doped samples[20] and with that of Chen et al. for the Al-doped samples[1]. Figure 7 shows a good correlation between the measured change in carrier density and that predicted by the VEC model. This implies that the structural changes occurring during annealing result in a reduction of the charge carrier density by $20-30 \%$, which is of the same order of magnitude as the decrease in electrical conductivity shown in Figure 3. Thus, the charge carrier mobility was not significantly influenced by the annealing step, and microcrack formation and/or development are likely not responsible for the change in the electrical properties (hypothesis 1). Additional if cracks would appear or develop during annealing one would not expect quantitatively reproducible measurements of the resistivity change. Instead, our measurements support hypothesis 2 , i.e. the reduction of the electrical resistivity is the result of loss of charge carriers due to structural changes.

As a final remark we note that in a rigid band picture the reduction in charge carrier density upon annealing will shift the electrochemical potential towards the gap and with that also the Seebeck coefficient is expected to significantly increase (see Appendix). However this is not the case in our measurements. This observation is consistent with the measurements of Chen et al., where Al doping increased the charge carrier density by up to $20 \%$ but the Seebeck coefficient was only weakly affected [1]. We take this as a clear hint on a change of the band structure close to the gap withchanging carrier density. With increased V content we observe a better accordance of the transport properties to a model with higher effective mass which points to flatter bands and, with that a weaker change of the electrochemical potential with carrier density. Clearly, more research is needed to study the effects of the carrier concentration and of the crystal structure ( $\gamma$ value) on the band structure and occupancy close to the chemical potential and understand how these parameters influence the Seebeck coefficient of HMS materials.

\subsection{Thermoelectric performance}

We now focus on the thermoelectric performance of the HMS materials. Based on the results discussed in the previous section we chose to compare the thermoelectric properties of the materials after annealing for 10 hours at $600^{\circ} \mathrm{C}$ in air in order to reduce the effect of high temperature instabilities and compare the materials on a fairer basis. The low temperature Hall measurements and the high temperature thermoelectric properties are reported in figure 8. Note that the Hall data, the high temperature resistivity and Seebeck coefficient data reported in figure 8 , and the electrical properties reported in figure 1 and figure 3 agree within measurement error even though different measurement setups were used. Despite our efforts to reduce the instabilities by pre-annealing, the electrical resistivity data between room temperature and $700^{\circ} \mathrm{C}$ clearly show that the electrical properties are not stabilized for all materials: the resistivity still increases during the measurement for Ge and Al-doped HMS. This is consistent with our observation that the resistivity still increases after 10 hours at $600{ }^{\circ} \mathrm{C}$ (figure 1 and figure 3 ) for these materials. However, the 3\%Re-HMS show a resistivity decrease during the measurement: this contradicts our data in figure 1 and figure 3 . The hysteresis behavior of the resistivity of this material could be related to a slow kinetics of $\gamma$ change following the temperature-time profile of the measurement with a delay. If for $2 \% \mathrm{~V}$-HMS $\gamma(T)$ behaves qualitatively as the undoped sample of Kikuchi et al.[13] above $600{ }^{\circ} \mathrm{C}$ (figure 6), delayed $\gamma$ changes would result in a hysteresis in the carrier density (hence in the resistivity) consistent with the measurement. The $4 \% \mathrm{~V}$-HMS is stable during the high temperature measurements, which is consistent with the stability study.

Figure 8 shows that the V-doped HMS samples have significantly different electrical properties to the $\mathrm{Al}, \mathrm{Ge}$ and Re-doped materials. The higher hole concentration of $2 \% \mathrm{~V}$ and $4 \% \mathrm{~V}$ HMS compared to the other materials is the result of the stability of $\mathrm{V}$ in the structure, while the other materials seem to stabilize at a carrier concentration of about $1.64 \times 10^{27} \mathrm{~m}^{-3}$ at $300 \mathrm{~K}$ (figure 8). Perhaps unexpected is the observation that the carrier concentration of the V-doped HMS materials increases with increasing temperature: unless very close to $0 \mathrm{~K}$ p-type thermoelectric materials typically possess a filled valence band and one does not expect this behaviour. This may be the result 
of impurity levels in the band gap. The Hall measurements also show a significantly lower charge carrier mobility for the V-doped materials, and a different temperature dependence. The $\mathrm{Al}, \mathrm{Ge}$ and Re-doped materials all have the typical temperature dependence $\mu_{L} \propto T^{1.5}$ demonstrating that latticephonon scattering is the dominant limiting factor for carrier transport, as also concluded from measurements on undoped or lightly doped HMS materials by other authors[1,2]. The mobility of the V-doped materials is nearly an order of magnitude lower and varies with temperature as $\mu \propto T^{0.5}$. Contrary to the $\mathrm{Al}, \mathrm{Ge}$ and $\mathrm{Re}$-doped materials for which the resistivity vanishes when the temperature tends to $0 \mathrm{~K}$ (confirming the phonon-limiting behaviour of the electrical transport), the resistivity of the V-doped materials remains significant at temperatures close to $0 \mathrm{~K}$. Overall the temperature dependence of the Seebeck coefficient and of the electrical resistivity is significantly less strong for the V-doped materials. This is precisely the nearly temperature-independent resistivity that confers the superior figure of merit for the $4 \% \mathrm{~V}$-HMS material. Finally we note that the charge carrier concentration and mobility of the Ge and $\mathrm{V}$-doped samples have comparable value as that of arcmelted samples with similar substitution levels recently published by Miyazaki et al. [20]. The lower mobility of V-doped HMS materials, their different temperature dependence and higher charge carrier density suggests that ionized impurity scattering is an additional limitation to the transport properties of these materials compared to the $\mathrm{Al}, \mathrm{Ge}$, and Re-doped materials. In general, the scattering time dominates the temperature dependence of the mobility $(\mu(T) \propto(e / m) \tau(\mathrm{T})$ where $e$ and $m$ are the electron charge and mass, respectively). The scattering time for ionized impurities scales as $\tau_{i} \propto T^{1.5}$, and that of phonons as $\tau_{L} \propto T^{1.5}$. When both effects occur simultaneously the total scattering time $\tau$ will follow Matthiessen's rule as:

$$
\frac{1}{\tau}=\frac{1}{\tau_{L}}+\frac{1}{\tau_{i}}
$$

Equation (3) implies that in the case of mixed scattering the temperature exponent of $\tau$ varies between -1.5 and 1.5, which is the case for the V-doped samples. Based on these observations we choose to use the mixed scattering single parabolic band model of Fistul to describe the behaviour of the V-doped materials[39]. The mobility depends on both the scattering time for acoustic vibrations $\tau_{L}$ $=\tau_{O L} \varepsilon^{-1 / 2}$ and the scattering time for ionized impurities $\tau_{i}=\tau_{0 i} \varepsilon^{3 / 2}$ where $\varepsilon$ is the reduced carrier energy. Using equation (3) the total scattering time can be written as $\tau=\tau_{o L} \varepsilon^{3 / 2} /\left(\varepsilon^{2}+a^{2}\right)$, where the scattering index $a=\sqrt{ } \tau_{o L} / \tau_{o i}$ is a measure of the strength of ionized impurity scattering relative to acoustic phonon scattering. In this framework the electrical properties can be written as follows:

$$
\begin{aligned}
& \sigma(\eta, a)=\frac{8 \pi m^{*} \frac{1}{2} e^{2}}{3 h^{3}}\left(2 k_{B} T\right)^{\frac{3}{2}} \tau_{0, L} \Phi_{3}(\eta, a) \\
& S(\eta, a)=\frac{k_{B}}{e}\left[\frac{\Phi_{4}(\eta, a)}{\Phi_{3}(\eta, a)}-\eta\right] \\
& {[p]=4 \pi\left(\frac{2 m^{*} k_{B} T}{h^{2}}\right)^{\frac{3}{2}} F_{\frac{1}{2}}(\eta)} \\
& \mu=\frac{2 e}{3 m^{*}} \tau_{0, L} \frac{\Phi_{3}(\eta, a)}{F_{1}(\eta)} \\
& \Phi_{n}(\eta, a)=\int_{0}^{\infty} \frac{\varepsilon^{n} \exp (\varepsilon-\eta)}{\left(\varepsilon^{2}+a^{2}\right)[1+\exp (\varepsilon-\eta)]^{2}} d \varepsilon
\end{aligned}
$$

Here $\mathrm{F}_{1 / 2}(\eta)$ is the Fermi integral of order $1 / 2 . \eta, m^{*}, k_{B}, h$ are the reduced Fermi energy, the density of states effective mass, Boltzmann coefficient and Planck constant respectively. It is assumed that the density of states effective mass is equal to the mobility effective mass. This model is an extension of the more commonly used single parabolic band model where only acoustic phonon scattering is considered[40] (limiting case $a=0$ ). This mixed scattering model has also been used by several authors to describe the superior properties of highly substituted skutterudites[41,42]. We solved the set of equations [4]-[7] numerically for two sets of $\left\{m^{*} ; a\right\}$ to illustrate the differences between the $4 \% \mathrm{~V}-\mathrm{HMS}$ and the Re, Ge and $\mathrm{Al}$ substituted materials. We assumed that $[p]$ is temperatureindependent and chose the value measured at room temperature to estimate $\eta(T)$ (equation (6)). First, we fit the model to the data of the Re, Ge and Al-doped samples using $a=0\left(\tau_{i} \rightarrow \infty\right.$ negligible ionized impurity scattering) and $[p]=1.64 \times 10^{27} \mathrm{~m}^{-3}$ (as measured at room temperature). Using the Seebeck coefficient data and equation [5] we obtain $m^{*}=7.5 m_{0}$ from equation (6), a value similar to 
that previously published[1]. $\tau_{o L}$ is estimated by fitting the mobility data, resulting in $\tau=\tau_{o L}=19.2 \mathrm{fs}$ at room temperature. The same value of $\tau_{o L}$ is used for $\mathrm{V}$-doped samples since there is a priori no reason that phonon scattering affects the electrical transport properties differently for V-doped samples and $\mathrm{Al}, \mathrm{Ge}$ and Re-doped materials. However, to reproduce the specific features of the data of Vdoped HMS it is necessary to introduce ionized impurity scattering (i.e. $a>0$ ). Using $m^{*}=10.5 m_{0}$ and $a(300 \mathrm{~K})=4.3\left(\tau_{i}(300 \mathrm{~K})=1 \mathrm{fs}\right)$, the model reproduces the room temperature data of $4 \% \mathrm{~V}$-HMS well. Figure 8 shows that the essential differences between the V-doped samples and the $\mathrm{Al}, \mathrm{Ge}$ and Re-doped samples are well described by introducing ionized impurity scattering. Especially the significant differences in the temperature dependence of the mobility, the low temperature resistivity data and the high temperature Seebeck coefficient data (at temperatures below about $450^{\circ} \mathrm{C}$ ) are well reproduced: the mobility scales as $\mu \propto T^{0.5}$, the resistivity does not vanish towards $0 \mathrm{~K}$, and the temperature dependence of $S(T)$ is less pronounced. However, while the model qualitatively predicts that the resistivity of V-doped materials becomes lower than that of the other samples at high temperature, there is a significant discrepancy between the values predicted by the model and the measurements.

The thermal conductivity of $4 \% \mathrm{~V}$-HMS is significantly lower than that of the other samples. Since this material also has a lower electrical resistivity, the electrical contribution to the thermal conductivity should be higher: this means that the lattice contribution to the thermal conductivity overcompensates the higher electrical contribution. Although this effect should be studied in more detail, we hypothesize that this may be the result of an alloy scattering effect. Overall, the significantly higher $Z T$ of $4 \% \mathrm{~V}-\mathrm{HMS}$ is the result of the improved power factor and lower thermal conductivity. $Z T_{\max }=0.52$ is estimated for this material. Most importantly, this material is also stable at high temperature, in contrast to the $\mathrm{Al}, \mathrm{Ge}$, Re-doped samples and the $2 \% \mathrm{~V}$-HMS material.

\section{Conclusions}

On the basis that the performance of thermoelectric materials should remain stable under relevant device temperature conditions, we have used several procedures to measure the structural and thermoelectric stability of HMS materials substituted with different elements. We demonstrate that most HMS materials prepared by the RGS technique have a temperature-dependent $\gamma$ factor and are structurally and electrically unstable at $600^{\circ} \mathrm{C}$. Specifically, structural changes result in the formation of $\mathrm{MnSi}$ precipitates and reduction of the charge carrier concentration of a magnitude comparable with the electrical conductivity decrease. Therefore we conclude that the structural changes are responsible for the unstability. By substituting $4 \%$ of $\mathrm{Mn}$ by $\mathrm{V}$ the $\gamma$ factor then becomes temperature independent in the range of interest, resulting in the prevention of $\mathrm{MnSi}$ precipitate formation and stable electrical properties when exposed to $600^{\circ} \mathrm{C}$ for up to $45 \mathrm{~h}$. As a topic for future research, it would be interesting to confirm the structural stability of this material up to the melting point.

The 4\%V-HMS material also exhibits the highest $Z T$. This is in part due to its thermal stability, but also because ionized impurity scattering effects mitigate the reduction of the Seebeck coefficient due to the higher charge carrier concentration. In combination with a lowered thermal conductivity, the estimated $Z T$ of this material reaches state-of-the-art values.

\section{Appendix}

For example, taking the Hall carrier concentration data for 0.45\%Al-HMS (figure 7) and using the single parabolic band model at $300 \mathrm{~K}$ (equations (4)-(8) with $a=0, m^{*}=7.5 m_{0}$ ), one finds that the carrier concentration change during the $10 \mathrm{~h}$ annealing step corresponds to a decrease in electrical conductivity from $90 \times 10^{4} \mathrm{~S} / \mathrm{m}$ to $70 \times 10^{4} \mathrm{~S} / \mathrm{m}(-22 \%$ - note that the absolute values fit the data of figure 3 fairly well) and to an increase of the Seebeck coefficient from $83 \mu \mathrm{V} / \mathrm{K}$ to $101 \mu \mathrm{V} / \mathrm{K}(+22 \%$, whereas measurements show a change from $110 \mu \mathrm{V} / \mathrm{K}$ to $100 \mu \mathrm{V} / \mathrm{K}$, i.e. $-9 \%$ ).

\section{Acknowledgements}


This work is partly financially supported by Rijksdienst voor Ondernemend Nederland (RVO) under project HEAT (ID: IK14062).

The authors would like to thank Przemyslaw Blaschkewitz for his help with the measurement of transport properties done at DLR.

\section{References}

[1] X. Chen, A. Weathers, D. Salta, L. Zhang, J. Zhou, J.B. Goodenough, L. Shi, Effects of $(\mathrm{Al}, \mathrm{Ge})$ double doping on the thermoelectric properties of higher manganese silicides, J. Appl. Phys. 114 (2013) 173705. https://doi.org/10.1063/1.4828731.

[2] M. Fedorov, V. Zaitsev, Thermoelectrics of Transition Metal Silicides, in: Thermoelectr. Handb., CRC Press, 2005: pp. 31-1-31-20. https://doi.org/10.1201/9781420038903.ch31.

[3] T. Zhu, Y. Liu, C. Fu, J.P. Heremans, J.G. Snyder, X. Zhao, Compromise and Synergy in High-Efficiency Thermoelectric Materials, Adv. Mater. 29 (2017) 1605884.

https://doi.org/10.1002/adma.201605884.

[4] J. de Boor, T. Dasgupta, U. Saparamadu, E. Müller, Z.F. Ren, Recent progress in p-type thermoelectric magnesium silicide based solid solutions, Mater. Today Energy. 4 (2017) 105121. https://doi.org/10.1016/j.mtener.2017.04.002.

[5] G. Skomedal, L. Holmgren, H. Middleton, I.S. Eremin, G.N. Isachenko, M. Jaegle, K. Tarantik, N. Vlachos, M. Manoli, T. Kyratsi, D. Berthebaud, N.Y. Dao Truong, F. Gascoin, Design, assembly and characterization of silicide-based thermoelectric modules, Energy Convers. Manag. 110 (2016) 13-21. https://doi.org/10.1016/j.enconman.2015.11.068.

[6] A. Yamamoto, S. Ghodke, H. Miyazaki, M. Inukai, Y. Nishino, M. Matsunami, T. Takeuchi, Thermoelectric properties of supersaturated Re solid solution of higher manganese silicides, Jpn. J. Appl. Phys. 55 (2016) 020301. https://doi.org/10.7567/JJAP.55.020301.

[7] S.C. Ghodke, Effect of grain boundaries and partial substitution of transition metals on thermoelectric properties of higher manganese silicide, $\mathrm{PhD}$ thesis, Nagoya University, 2016.

[8] S. Ghodke, N. Hiroishi, A. Yamamoto, H. Ikuta, M. Matsunami, T. Takeuchi, Enhanced Thermoelectric Properties of W- and Fe-Substituted MnSi $\gamma$, J. Electron. Mater. 45 (2016) 5279-5284. https://doi.org/10.1007/s11664-016-4688-x.

[9] Y. Miyazaki, Superspace Group Approach to the Crystal Structure of Thermoelectric Higher Manganese Silicides MnSi $\gamma$, in: Neutron Diffr., 2012: pp. 231-242. https://doi.org/10.5772/36666.

[10] L. Akselrud, R. Cardoso Gil, M. Wagner-Reetz, Y. Grin, Disorder in the composite crystal structure of the manganese "disilicide" MnSi1.73 from powder X-ray diffraction data, Acta Crystallogr. Sect. B Struct. Sci. Cryst. Eng. Mater. 71 (2015) 707-712. https://doi.org/10.1107/S2052520615019757.

[11] D.C. Fredrickson, S. Lee, R. Hoffmann, The Nowotny chimney ladder phases: Whence the 14 electron rule?, Inorg. Chem. 43 (2004) 6159-6167. https://doi.org/10.1021/ic049897h.

[12] D.C. Fredrickson, S. Lee, R. Hoffmann, J. Lin, The Nowotny chimney ladder phases: Following the cpseudo clue toward an explanation of the 14 electron rule, Inorg. Chem. 43 (2004) 6151-6158. https://doi.org/10.1021/ic049427n.

[13] Y. Kikuchi, T. Nakajo, K. Hayashi, Y. Miyazaki, High temperature X-ray diffraction study on incommensurate composite crystal $\mathrm{MnSi}-(3+1)$-dimensional superspace approach, J. Alloys Compd. 616 (2014) 263-267. https://doi.org/10.1016/j.jallcom.2014.07.106.

[14] Y. Miyazaki, D. Igarashi, K. Hayashi, T. Kajitani, K. Yubuta, Modulated crystal structure of 
chimney-ladder higher manganese silicides MnSi $(\gamma \sim 1.74)$, Phys. Rev. B - Condens. Matter Mater. Phys. 78 (2008) 1-8. https://doi.org/10.1103/PhysRevB.78.214104.

[15] I. Kawasumi, M. Sakata, I. Nishida, K. Masumoto, Crystal growth of manganese silicide, MnSi 1.73 and semiconducting properties of Mn15Si26, J. Mater. Sci. 16 (1981) 355-366. https://doi.org/10.1007/BF00738624.

[16] R. De Ridder, S. Amelinckx, The structure of defect manganese silicides, Mater. Res. Bull. 6 (1971) 1223-1234. https://doi.org/10.1016/0025-5408(71)90058-4.

[17] H.Q. Ye, S. Amelinckx, High-resolution electron microscopic study of manganese silicides MnSi2-x, J. Solid State Chem. 61 (1986) 8-39. https://doi.org/10.1016/0022-4596(86)900034.

[18] S. Vivès, Microstructure control and transport properties of incommensurate manganese silicide based alloys for thermoelectricity, PhD thesis, Universite de Bordeaux, 2015.

[19] D.Y.N. Truong, Thermoelectric Properties of Higher Manganese Silicides, PhD thesis, University of Waterloo, Waterloo, Ontario, Canada, 2015.

[20] H. Hamada, Y. Kikuchi, K. Hayashi, Y. Miyazaki, Crystal Structure and Thermoelectric Properties of the Incommensurate Chimney-Ladder Compound VGe $\gamma(\gamma \sim 1.82)$, J. Electron. Mater. 45 (2016) 1365-1368. https://doi.org/10.1007/s11664-015-4036-6.

[21] A. Allam, P. Boulet, C. a. Nunes, J. Sopousek, P. Broz, M.C. Record, Phase transformations in Higher Manganese Silicides, J. Alloys Compd. 551 (2013) 30-36.

https://doi.org/10.1016/j.jallcom.2012.10.016.

[22] I. Aoyama, M.I. Fedorov, V.K. Zaitsev, F.Y. Solomkin, I.S. Eremin, A.Y. Samunin, M. Mukoujima, S. Sano, T. Tsuji, Effects of Ge Doping on Micromorphology of MnSi in MnSi $\sim 1.7$ and on Their Thermoelectric Transport Properties, Jpn. J. Appl. Phys. 44 (2005) 85628570. https://doi.org/10.1143/JJAP.44.8562.

[23] E.I. Suvorova, V. V. Klechkovskaya, Precipitates of MnSi cubic phase in tetragonal Mn4Si7 crystal, Crystallogr. Reports. 58 (2013) 854-861. https://doi.org/10.1134/S1063774513060229.

[24] A.S. Orekhov, E.I. Suvorova, Study of doped higher manganese silicides crystals by transmission electron diffraction and electron backscatter diffraction, Crystallogr. Reports. 59 (2014) 78-87. https://doi.org/10.1134/S106377451401009X.

[25] L.D. Ivanova, A.A. Baikov, Higher Manganese Silicide Based Materials, J. Thermoelectr. 3 (2009) 60-66.

[26] A.J. Zhou, X.B. Zhao, T.J. Zhu, S.H. Yang, T. Dasgupta, C. Stiewe, R. Hassdorf, E. Mueller, Microstructure and thermoelectric properties of SiGe-added higher manganese silicides, Mater. Chem. Phys. 124 (2010) 1001-1005. https://doi.org/10.1016/j.matchemphys.2010.08.017.

[27] Y. Miyazaki, H. Hamada, K. Hayashi, K. Yubuta, Crystal Structure and Thermoelectric Properties of Lightly Vanadium-Substituted Higher Manganese Silicides (Mn1-xVx)Si $)^{\text {, J. }}$ Electron. Mater. 46 (2017) 2705-2709. https://doi.org/10.1007/s11664-016-4937-z.

[28] Y. Miyazaki, H. Hamada, H. Nagai, K. Hayashi, Crystal structure and thermoelectric properties of lightly substituted higher manganese silicides, Materials (Basel). 11 (2018) 926. https://doi.org/10.3390/ma11060926.

[29] M. Apel, D. Franke, I. Steinbach, Simulation of the crystallisation of silicon ribbons on substrate, in: Sol. Energy Mater. Sol. Cells, 2002: pp. 201-208. https://doi.org/10.1016/S09270248(01)00165-9.

[30] G. Hahn, A. Schönecker, New crystalline silicon ribbon materials for photovoltaics, J. Phys. Condens. Matter. 16 (2004) R1615-R1648. https://doi.org/10.1088/0953-8984/16/50/R03. 
[31] V. Petříček, V. Eigner, M. Dušek, A. Čejchan, Discontinuous modulation functions and their application for analysis of modulated structures with the computing system JANA2006, Zeitschrift Für Krist. - Cryst. Mater. 231 (2016). https://doi.org/10.1515/zkri-2015-1913.

[32] J. de Boor, C. Stiewe, P. Ziolkowski, T. Dasgupta, G. Karpinski, E. Lenz, F. Edler, E. Mueller, High-Temperature Measurement of Seebeck Coefficient and Electrical Conductivity, J. Electron. Mater. 42 (2013) 1711-1718. https://doi.org/10.1007/s11664-012-2404-z.

[33] J. de Boor, E. Müller, Data analysis for Seebeck coefficient measurements, Rev. Sci. Instrum. 84 (2013) 065102. https://doi.org/10.1063/1.4807697.

[34] Y. Miyazaki, Y. Saito, K. Hayashi, K. Yubuta, T. Kajitani, Preparation and Thermoelectric Properties of a Chimney-Ladder (Mn 1- x Fe x )Si $\gamma(\gamma \sim 1.7)$ Solid Solution, Jpn. J. Appl. Phys. 50 (2011) 035804. https://doi.org/10.1143/JJAP.50.035804.

[35] N. Krendelsberger, F. Weitzer, J.C. Schuster, On the constitution of the system Al-Mn-Si, Metall. Mater. Trans. A. 33 (2002) 3311-3319. https://doi.org/10.1007/s11661-002-0319-7.

[36] A. Yamamoto, H. Miyazaki, M. Inukai, Y. Nishino, T. Takeuchi, Thermoelectric properties of Al-Mn-Si C40 phase containing small amount of W or Ta, Jpn. J. Appl. Phys. 54 (2015) 071801. https://doi.org/10.7567/JJAP.54.071801.

[37] H.W. Kerr, W. Kurz, Solidification of peritectic alloys, Int. Mater. Rev. 41 (1996) 129-164. https://doi.org/10.1179/095066096790151231.

[38] T. Kojima, I. Nishida, T. Sakata, Crystal growth of Mn15Si26, J. Cryst. Growth. 47 (1979) 589-592. https://doi.org/10.1016/0022-0248(79)90144-1.

[39] V.I. Fistul, Heavily Doped Semiconductors, Plenum, New York, 1969.

[40] A.F. May;, G.J. Snyder, Introduction to Modeling Thermoelectric Transport at High Temperatures, in: D.M. Rowe (Ed.), Mater. Prep., Charact. Thermoelectr., CRC Press, 2012.

[41] S. Wang, J. Yang, L. Wu, P. Wei, W. Zhang, J. Yang, On Intensifying Carrier Impurity Scattering to Enhance Thermoelectric Performance in Cr-Doped Ce y Co 4 Sb 12, Adv. Funct. Mater. 25 (2015) 6660-6670. https://doi.org/10.1002/adfm.201502782.

[42] J.S. Dyck, W. Chen, C. Uher, L. Chen, X. Tang, T. Hirai, Thermoelectric properties of the n type filled skutterudite Ba0.3Co4Sb12 doped with Ni, J. Appl. Phys. 91 (2002) 3698-3705. https://doi.org/10.1063/1.1450036. 
Figures and captions
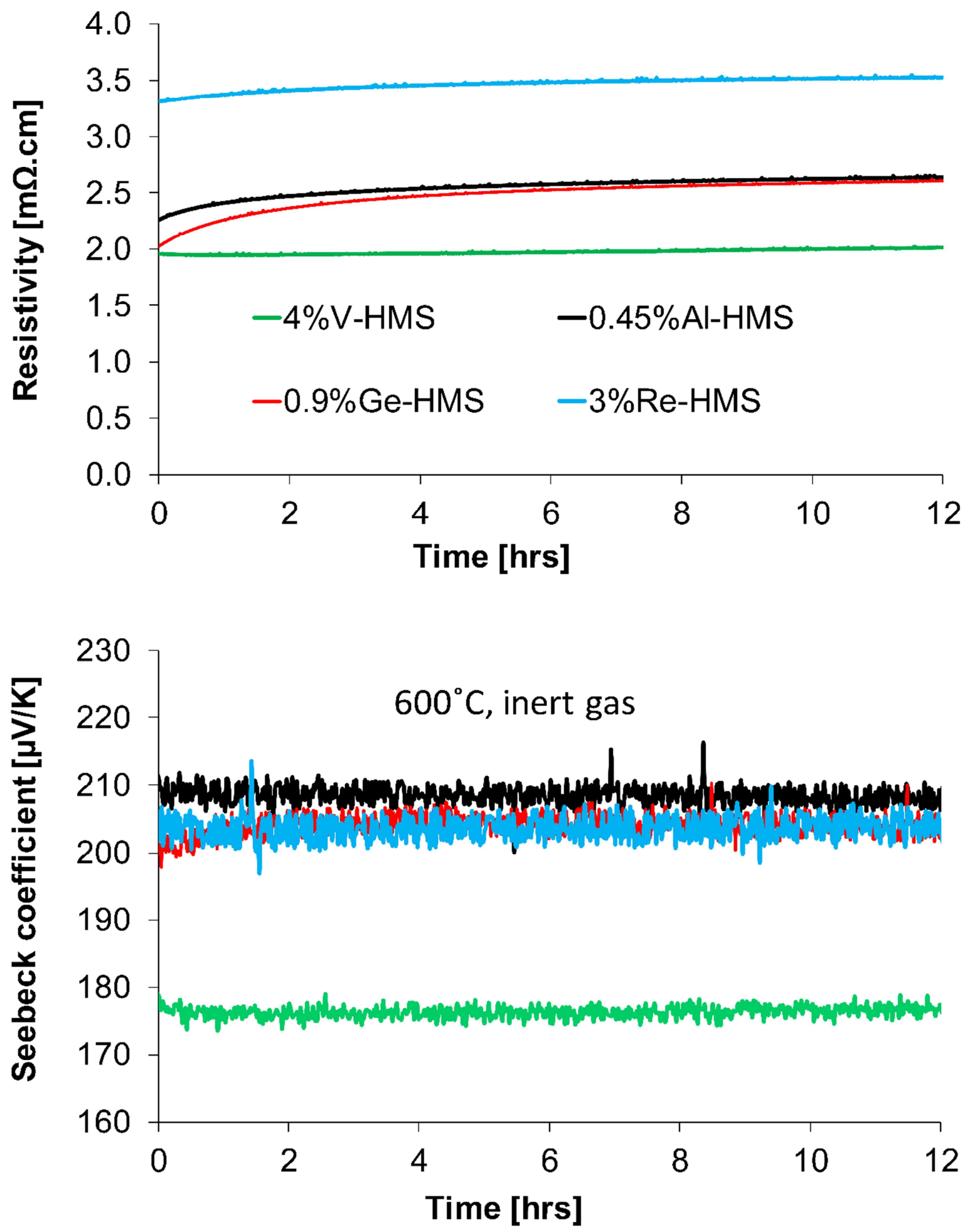

Figure 1. (Color Online) Seebeck coefficient and electrical resistivity as a function of time at high temperature $\left(600{ }^{\circ} \mathrm{C}\right)$ in inert gas (measurements Uni Groningen). The samples were as-solidified prior to the measurement. 
$3 \%$ Re-HMS Uni Groningen

$2 \% \mathrm{~V}-\mathrm{HMS}$ DLR

0.45\%Al-HMS Uni Groningen

- 4\%V-HMS CEA

x 4\%V-HMS Uni Twente

measurement sequence

r ------4

10h
$0.9 \% \mathrm{Ge}-\mathrm{HMS}$ Uni Groningen

$0.45 \%$ Al-HMS CEA

$4 \%$ V-HMS DLR

4\%V-HMS Uni Groningen

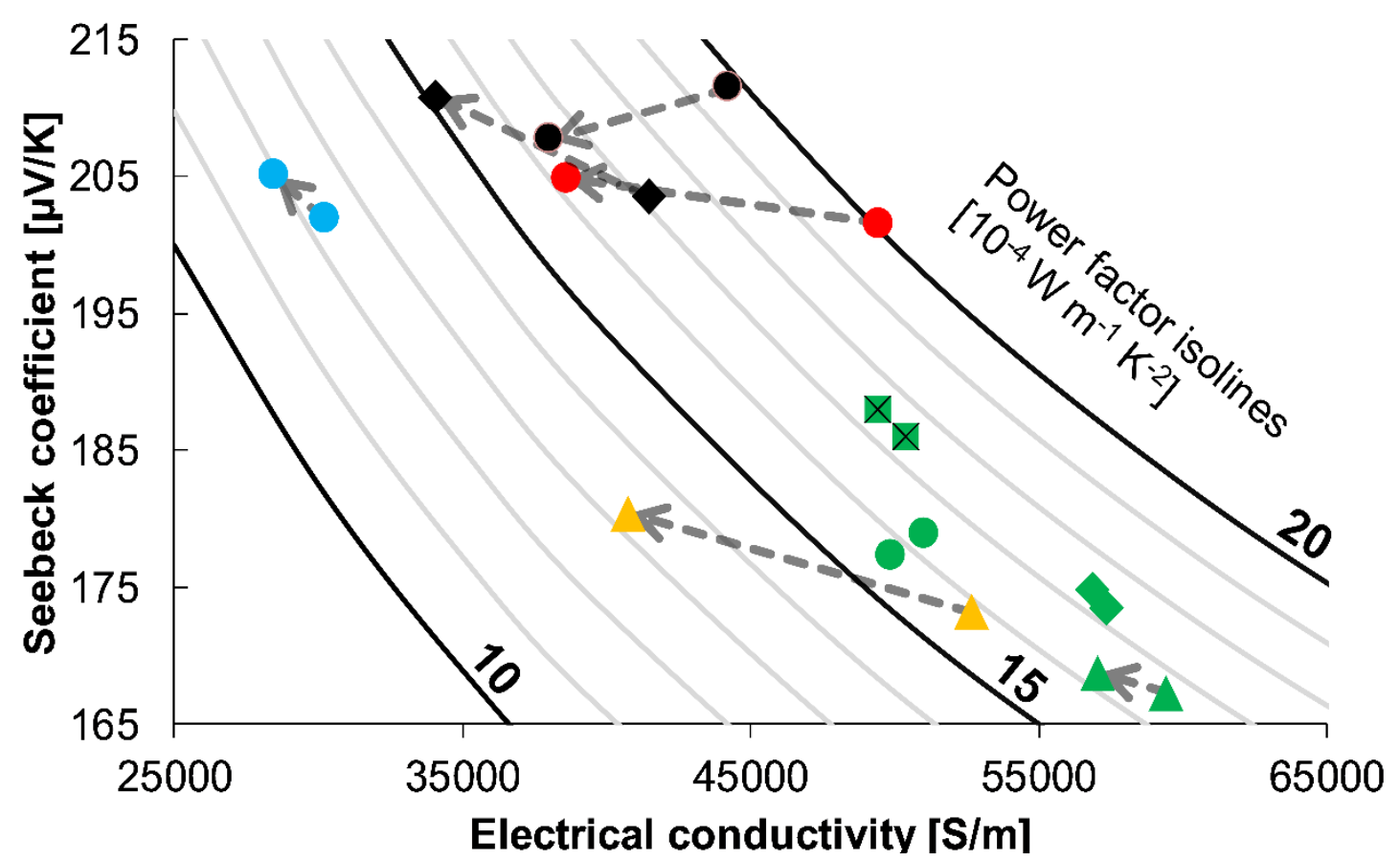

Figure 2. (Color Online) Seebeck coefficient and electrical conductivity of as-grown materials (points at start of arrows) and materials after $10 \mathrm{~h}$ annealing at $600^{\circ} \mathrm{C}$ (points at end of arrows). The curved lines are power factor isolines. Uni Groningen data are taken from Figure 1, CEA and Uni Twente data are taken from Supplementary information - Figure S1. DLR data (after $10 \mathrm{~h}$ ) are taken from figure 8 (average between temperature ramp up and ramp down). 
$0.9 \% \mathrm{Ge}-\mathrm{HMS} \quad 4 \% \mathrm{~V}-\mathrm{HMS}$

$2 \% \mathrm{~V}-\mathrm{HMS}$

$0.45 \% \mathrm{Al}-\mathrm{HMS}$

\section{$3 \% \operatorname{Re}-\mathrm{HMS}$}

measurement sequence

- $\leftarrow--\rightarrow---\rightarrow$

\section{$22^{\circ} \mathrm{C}-\mathrm{RGS}$}

$100 \mathrm{~h} \quad 10 \mathrm{~h}$ as solidified

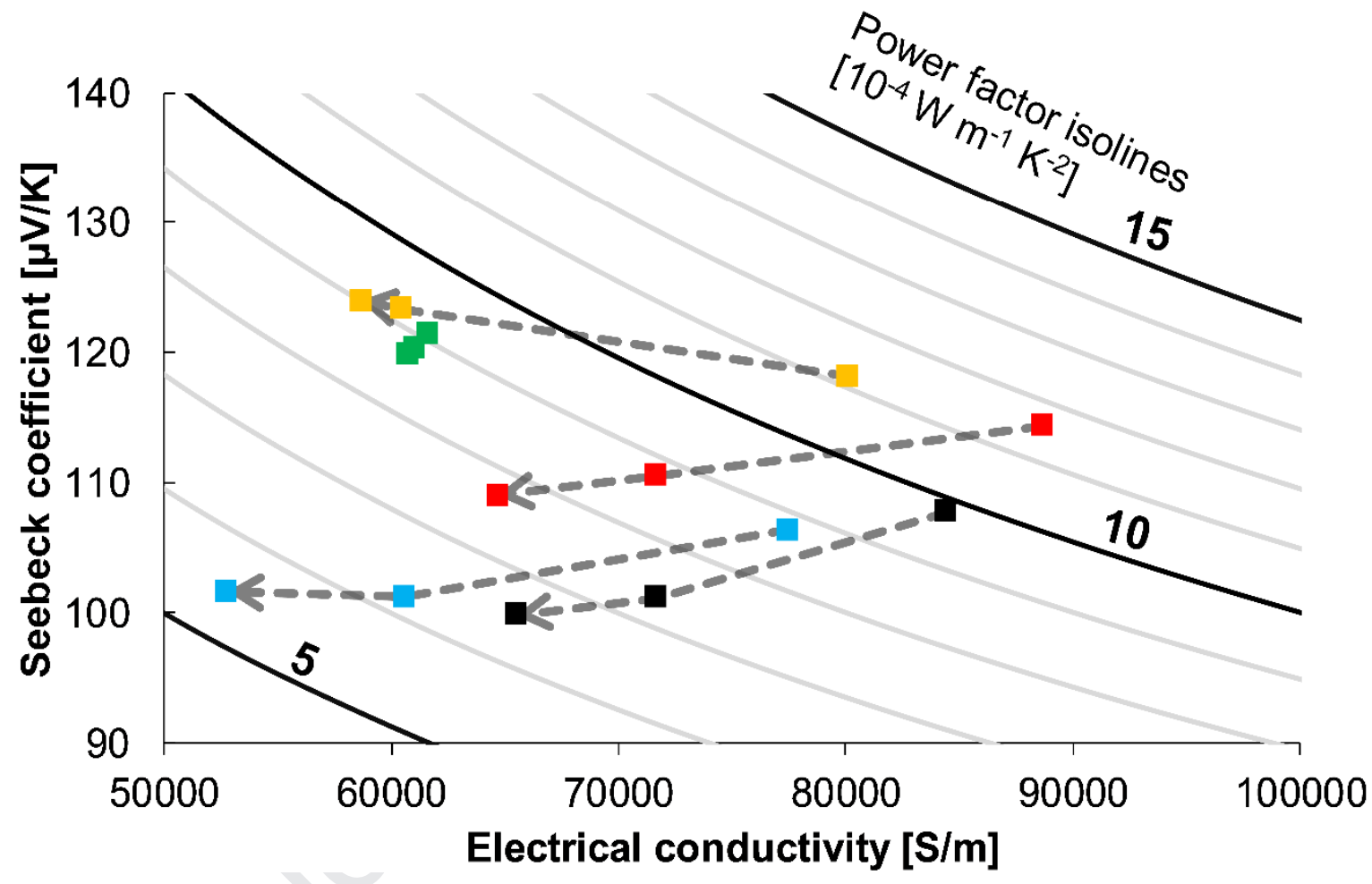

Figure 3. (Color Online) Seebeck coefficient and electrical conductivity of as-grown materials (points at start of arrows) and materials annealed for $10 \mathrm{~h}$ (second points along arrows) and $100 \mathrm{~h}$ (points at ends of arrows) in air at $600^{\circ} \mathrm{C}$. The curved lines are power factor isolines. Each data point corresponds to the average of 3 to 5 replicated measurements. The 3 measurement points for $4 \% \mathrm{~V}$ HMS overlap within the measurement error. 


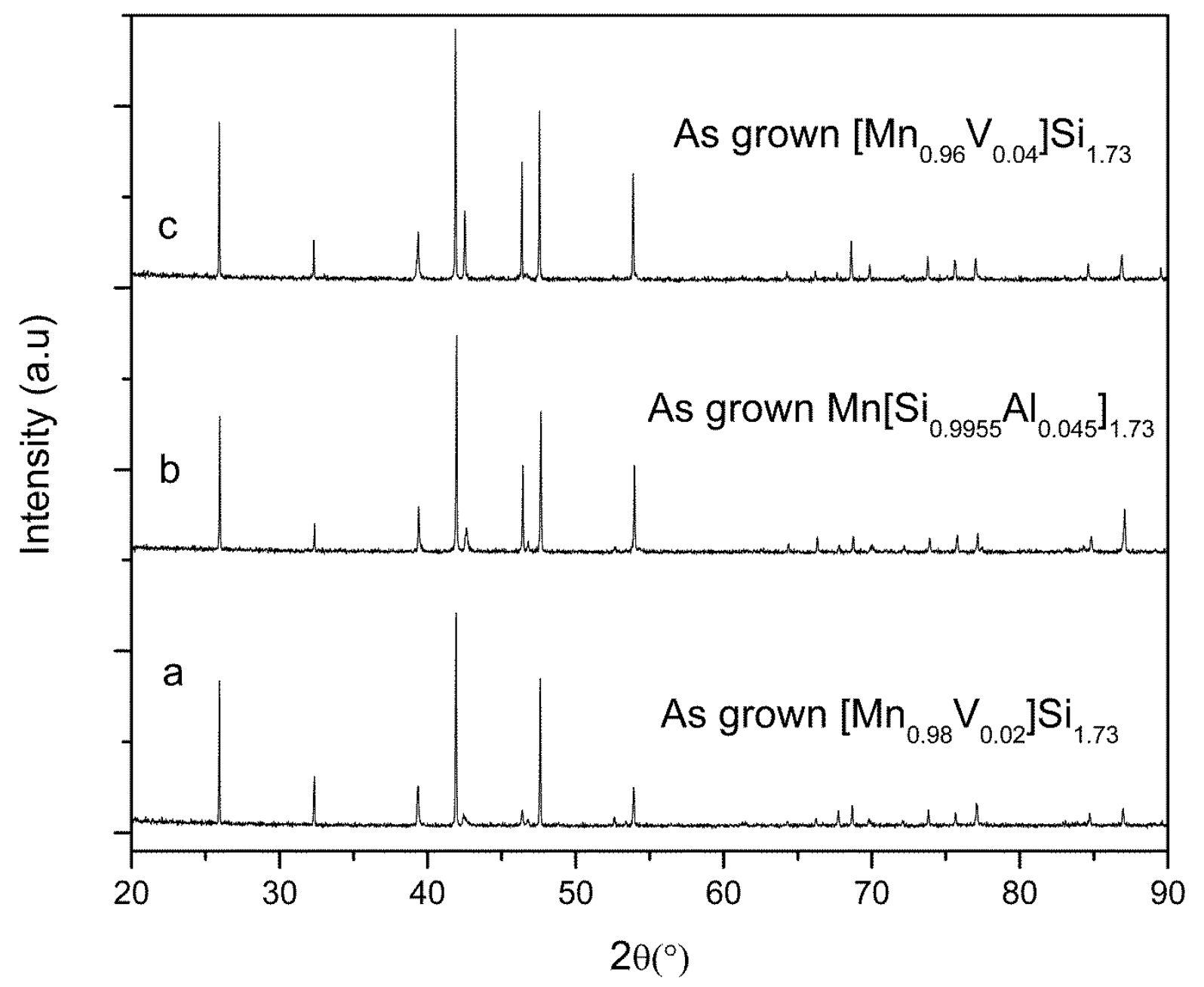

Figure 4. Room temperature XRD measurements of as-solidified (a) 2\%V-HMS, (b) 0.45\%Al-HMS, and (c) $4 \%$ V-HMS. 


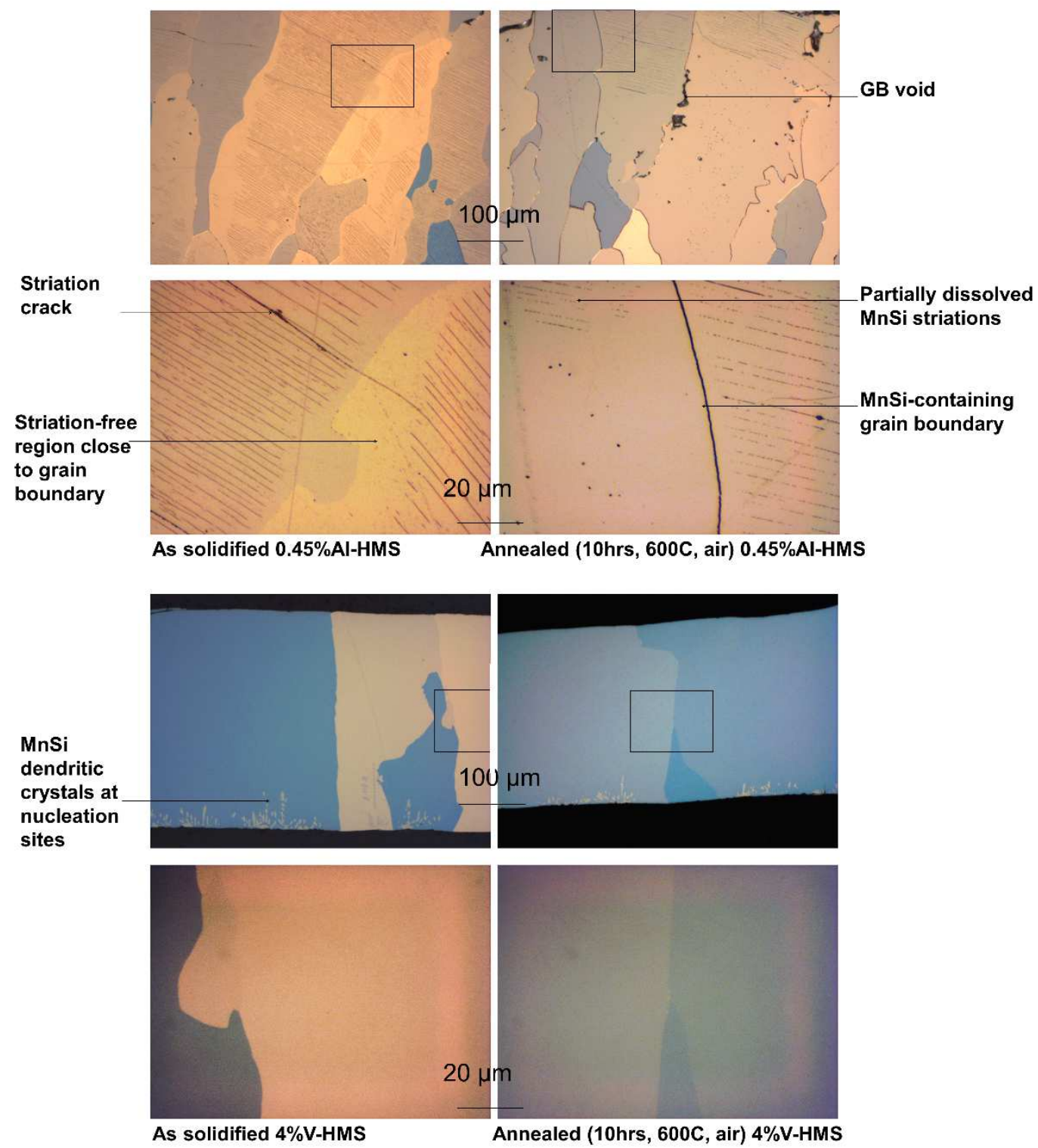

Figure 5. (Color Online) Optical microscopy images of polished cross sections of $0.45 \% \mathrm{Al}-\mathrm{HMS}$ (upper set) and 4\%V-HMS (lower set). The solidification direction is from bottom to top on all images. Pictures are taken under polarized light at low magnification (top) and high magnification (bottom - regions corresponding to the black inset frames above). As-solidified samples are shown on the left and annealed samples $\left(10 \mathrm{~h}\right.$ at $600{ }^{\circ} \mathrm{C}$ in air) on the right. All cross sections were submitted to a MnSi etch to reveal the presence of the MnSi phase. 


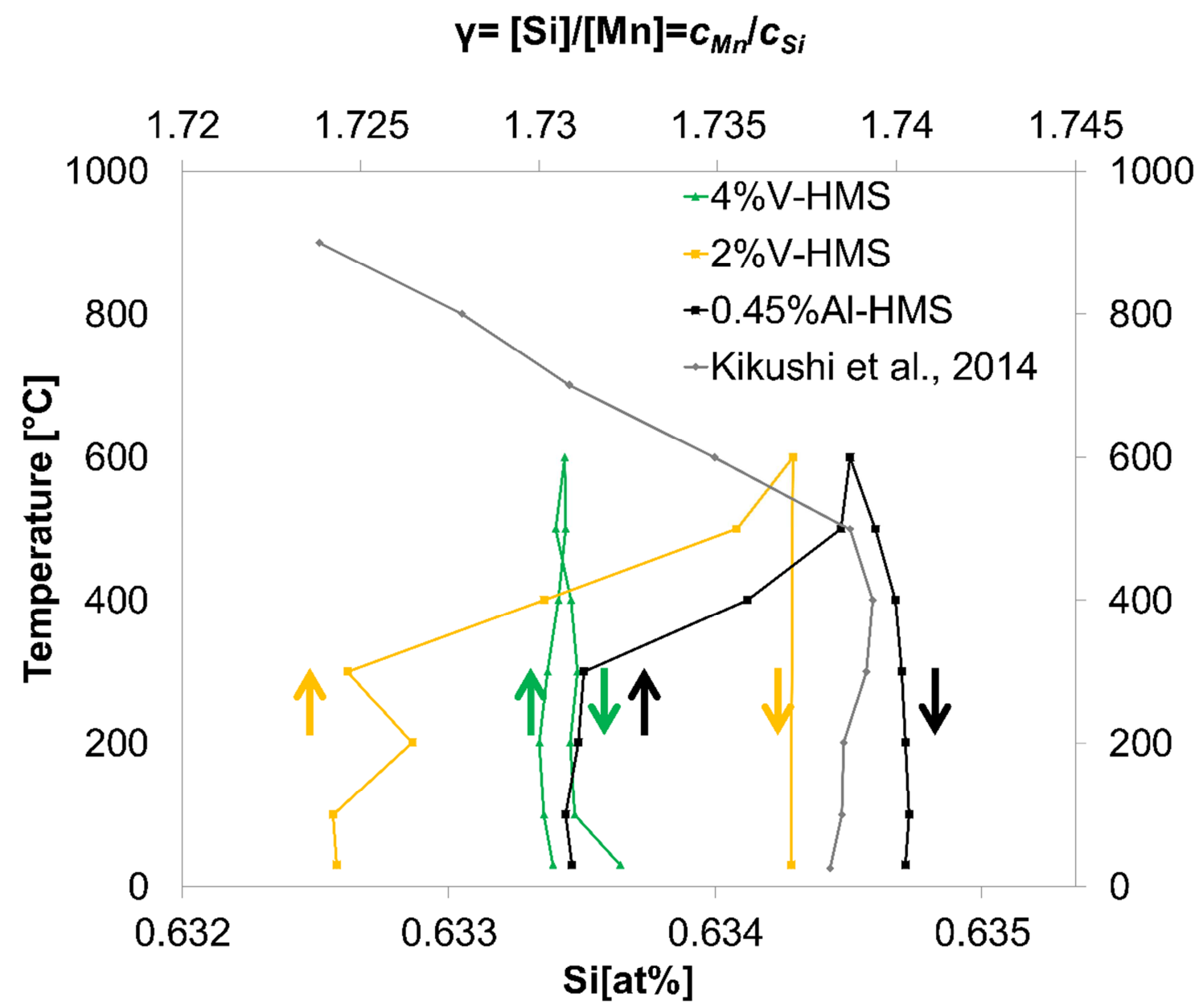

Figure 6. (Color Online) $\gamma(T)$ structural parameter for as-solidified HMS materials measured by HTXRD. The arrows show the evolution of $\gamma$ upon heating ( $\uparrow)$ and cooling $(\downarrow)$. The data of Kikuchi et al.[13] for undoped HMS prepared by arc melting is shown for comparison and discussion purposes. 


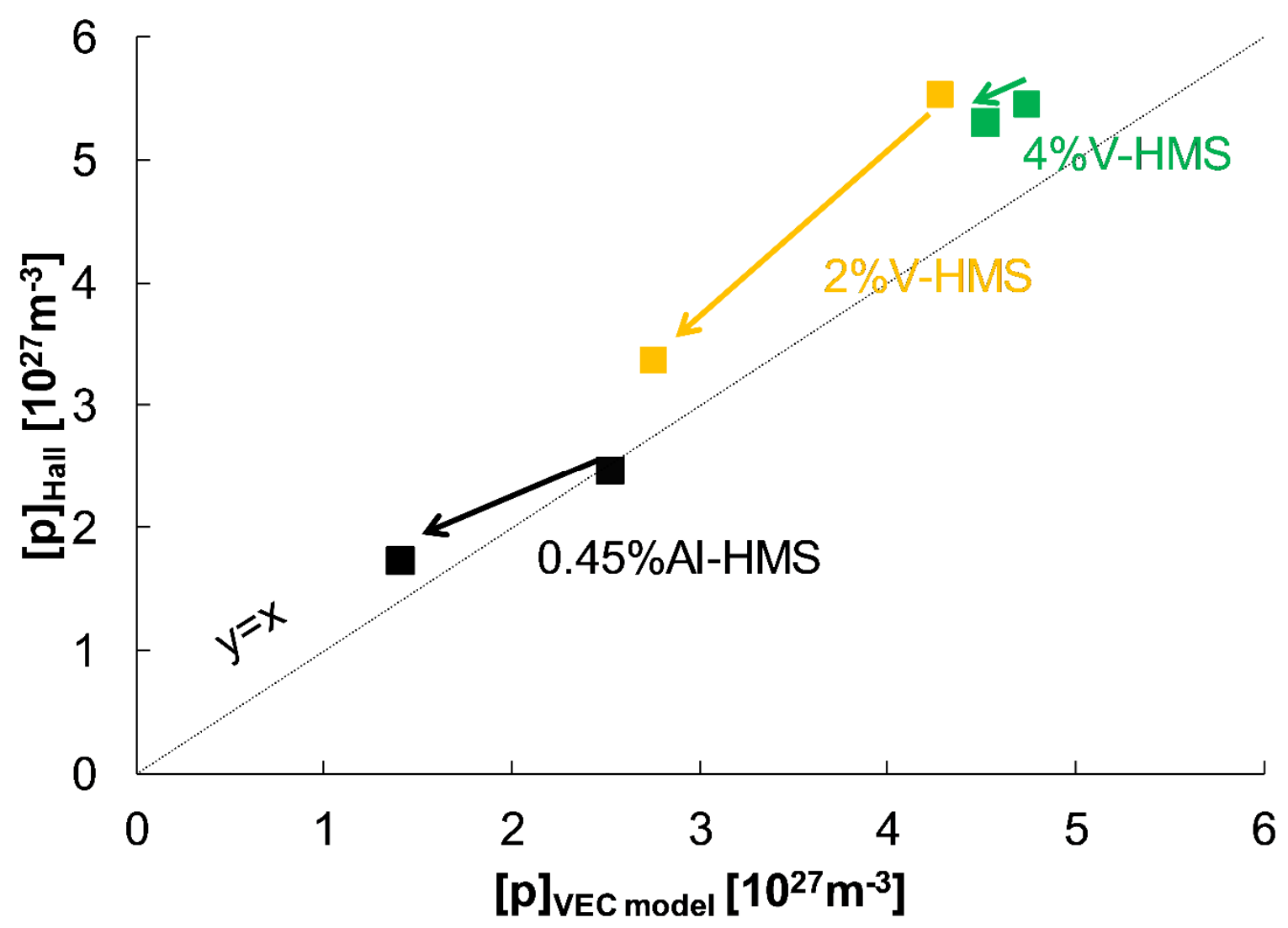

Figure 7. (Color Online) Effect of annealing on the charge carrier density of 0.45\%Al-HMS, 2\%VHMS, and 4\%V-HMS. Hall measurements (vertical axis) are compared to the carrier density estimated from the VEC model (horizontal axis). The straight line corresponds to $\mathrm{y}=\mathrm{x}$. 

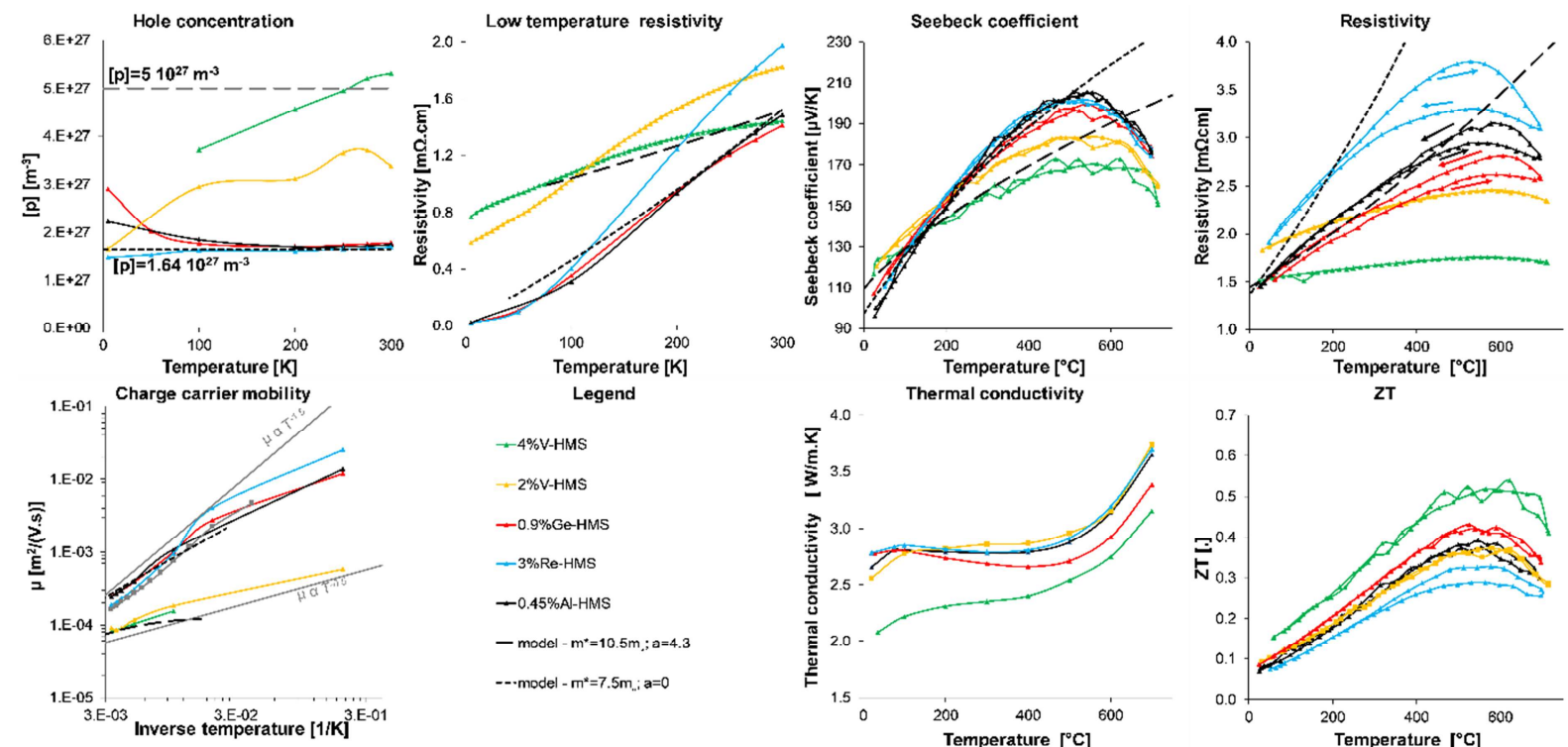

Figure 8. (Color Online) Low temperature (left) and high temperature (right) thermoelectric properties of HMS materials produced by the RGS process containing different substitution elements. All materials were annealed for 10 hours at $600{ }^{\circ} \mathrm{C}$ in air prior to the measurements. Materials doped with $\mathrm{Ge}, \mathrm{Al}$ and $\mathrm{Re}$ have not reached a stable state after this thermal treatment. The coloured arrows in the high temperature resistivity plots show the data acquisition sequence during heating and cooling. Dashed black lines are model results for different effective masses $m^{*}$ and scattering parameters $a$ at $300 \mathrm{~K}$. 
- Thermoelectric alloys $\mathrm{MnSi}_{\sim 1.74}$ were solidified by a new synthesis technique

- Long-term high temperature stability is characterized

- $\mathrm{Al}, \mathrm{Ge}$ and Re doped materials are neither structurally nor electrically stable

- $4 \% \mathrm{~V}$ substitution stabilizes the (micro)structure and electrical properties

- Vanadium-doped higher manganese silicide reaches state-of-the art $Z T=0.52$ 
Pierre-Yves Pichon: Conceptualization, Formal analysis, Investigation, Writing - Original Draft, Visualization, Supervision, Project administration

Pierre Berneron: Methodology, Validation, Formal analysis, Investigation, Data Curation, Writing - Review \& Editing, Visualization

Joshua Levinsky: Validation, Investigation, Data Curation

Arjan Burema: Validation, Investigation, Data Curation

Graeme Blake: Conceptualization, Methodology, Writing - Review \& Editing, Supervision

David Berthebaud: Methodology, Writing - Review \& Editing, Visualization

Stéphanie Gascoin: Investigation

Franck Gascoin: Conceptualization, Writing - Review \& Editing

Sylvie Hebert: Investigation, Data Curation, Writing - Review \& Editing

Jonas Amtsfeld: Validation, Data Curation, Investigation,

Tijmen Hommels: Validation, Data Curation, Investigation,

Mark Huijben: Conceptualization, Writing - Review \& Editing, Supervision

Johannes de Boor: Validation, Data Curation, Writing - Review \& Editing

Eckhard Müller: Writing - Review \& Editing

Christelle Navone: Validation, Data Curation, Writing - Review \& Editing

Axel Schönecker: Conceptualization, Supervision, Funding acquisition 


\section{Declaration of interests}

$\bigotimes$ The authors declare that they have no known competing financial interests or personal relationships that could have appeared to influence the work reported in this paper.

$\square$ The authors declare the following financial interests/personal relationships which may be considered as potential competing interests: 